\title{
Lagrangian dynamics of drift-wave turbulence
}

\author{
W.J.T. Bos ${ }^{\mathrm{a}}$, B. Kadoch ${ }^{\mathrm{b}}$, S. Neffaa ${ }^{\mathrm{b}}$ and K. Schneider ${ }^{\mathrm{b}}$ \\ ${ }^{\mathrm{a}}$ LMFA-CNRS, Ecole Centrale de Lyon - Université de Lyon, Ecully, France \\ b M2P2-CNRS \& CMI Universités d'Aix-Marseille, France
}

\begin{abstract}
The statistical properties of Lagrangian particle transport are investigated in dissipative drift-wave turbulence modelled by the Hasegawa-Wakatani system. By varying the adiabaticity parameter $c$, the flow regime can be modified from a hydrodynamic limit for $c=0$ to a geostrophic limit for $c \rightarrow \infty$. For $c$ of order unity the quasi-adiabatic regime is obtained, which might be relevant to describe the edge turbulence of fusion plasmas in tokamaks. This particularity of the model allows to study the change in dynamics when varying from one turbulent flow regime to another. By means of direct numerical simulation we consider four values for $c$ and show that the Lagrangian dynamics is most intermittent in the hydrodynamic regime, while the other regimes are not or only weakly intermittent of velocity increments, autocorrelation functions of velocity and acceleration, and structure functions. In both quasi-adiabatic and quasi-geostrophic regimes the PDFs of acceleration exhibit exponential tails. This behaviour is due to the pressure term in the acceleration and not a signature of intermittency.
\end{abstract}

\section{Introduction}

The understanding of the Lagrangian dynamics of fluid particles is of great practical interest, in particular for understanding and modeling turbulent transport and mixing. For a recent review we refer to [1]. One important application is the understanding of the dynamics of impurities in fusion plasmas. Indeed the confinement quality of a fusion plasma can be largely affected by the presence of heavy ions in the plasma. Their presence in the edge region might enhance the quality of the confinement by reinforcing transport barriers but the impurity accumulation in the core of the plasma might lead to heat loss by radiation, which decreases the confinement quality. A detailed understanding of impurity transport is therefore primordial in fusion plasma design and operation.

Transport in edge plasmas is largely anomalous, which means that it exceeds estimations based on Coulomb interactions only. This is now generally assumed to be due to turbulent electro-static drift-velocity fluctuations in the plasma edge e.g. $[2,3]$. To a certain extend, the impurities will follow the velocity fluctuations, which makes the Lagrangian description the most natural choice. It is important here to note that the impurity transport can be studied in two complementary ways. The first one is the passive scalar approach in which the impurity density in a fluid element is evaluated (see for example references [4,5]). This process is governed by an advection-diffusion equation for a passive scalar. An alternative approach consists of following individual marked fluid particles. These approaches are closely related but differ by the fact that the equation for the Lagrangian velocity contains a fluid-pressure term which is known to play a dominant role in the fluid particle acceleration. It is the second approach that we will apply in the present work.

The Lagrangian dynamics of fluid particles in NavierStokes turbulence have been investigated extensively since numerical simulations [6] and experimental methods [7] allow to follow individual fluid particles. In [6] it is shown that the Lagrangian dynamics display an intermittent behaviour. By intermittent we understand that the dynamics are not scale-invariant and thus do not display simple scaling behaviour as would be expected from dimensional analysis à la Kolmogorov 1941 [8]. In three dimensions it was shown that the Lagrangian intermittency is stronger than its Eulerian counterpart $[9,10]$. In two dimensions, it was recently shown that Lagrangian intermittency can exist even if it is completely absent in the Eulerian reference frame [11]. In wall bounded domains no-slip conditions can even enhance the Lagrangian intermittency significantly [12]. This intermittent behaviour constitutes a major challenge to modellers. Whereas the statistics of the complex dynamics of scale-invariant Lagrangian dynamics could be modelled by a Gaussian process, intermittency impedes this. Large fluctuations in the velocity increments and particle accelerations would be largely underestimated by such an approach. The main issue in the present work 
is therefore to characterize these dynamics in the case of plasma turbulence and to check whether it can, or not, be modelled by Gaussian processes.

In order to achieve this, we investigate the Lagrangian dynamics in a model for drift-wave turbulence in the tokamak edge, the Hasegawa-Wakatani two-field model [13]. Due to the strong toroidal magnetic field in tokamaks, the dynamics are close to two-dimensional. In the $2 \mathrm{D}$ version of the model, which we will consider here, two equations are solved. One equation governs the evolution of the vorticity, the other the advection of the plasma-density fluctuations. As we will see in the next section, the model is formally very similar to the dynamics of two-dimensional turbulence advecting a passive scalar. The difference is the presence of a coupling term related to the parallel (with respect to the toroidal magnetic field) dynamics of the plasma. An adjustable parameter $c$ in front of this term, related to the toroidal dynamics of the tokamak, allows to consider different regimes. For $c=0$ we obtain the hydrodynamic limit and for $c \rightarrow \infty$ one obtains the Charney-Hasegawa-Mima model $[14,15]$, frequently used in studies of the geostrophic dynamics in planetary atmospheres. An intermediate value for $c$ of order unity yields dynamics which are supposed to be close to tokamak edge-turbulence.

Eventually, the goal of the present work is two-fold. Not only will we answer the question how to practically model the Lagrangian dynamics of tokamak-relevant drift wave turbulence, we also show how the Lagrangian dynamics change when going from the hydrodynamic limit to the geostrophic limit.

The rest of the paper will be constructed as follows. In the next section we will write the equations and describe the numerical method. In section 3 we present some Eulerian characteristics of the different flows considered. In section 4 we present the Lagrangian statistics. These statistics and their interpretation constitute the main results of the present paper. Several observations are made which we summarize here.

(i) The Lagrangian acceleration, will, as soon as it is dominated by the pressure gradient, not be Gaussian. Indeed it was shown in [16] that the quadratic dependence of the pressure on the velocity can account for the exponential tails, and their occurrence is not an argument for 'intermittency' under any reasonable definition of the term. This argument holds also for the pressure gradient. Lagrangian velocity increment PDFs will therefore never be scale-invariant, but will always develop, at least, exponential tails.

(ii) Drift wave turbulence in the regimes $c=0.7,2$ and 4 is not intermittent. By increasing the adiabaticity parameter $c$ from $c=0.01$ upwards, the flaring tails of the acceleration PDF smoothly reduce to exponential tails.

(iii) Extended Self-Similarity (ESS) will for short times always yield behaviour in agreement with K41. It make the detection of the inertial range more difficult instead of simplifying it. This is not a new result,

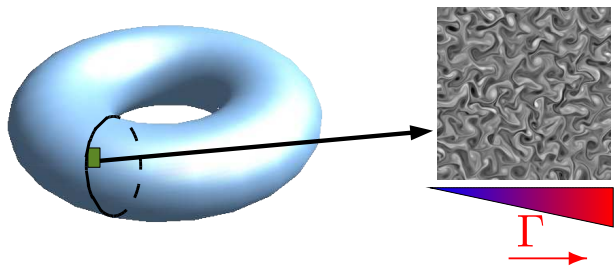

Fig. 1. Illustration of the slab geometry used in the present study.

but we want to stress this property of ESS, since it is sometimes ignored in investigations.

\section{Lagrangian dynamics of the Hasegawa-Wakatani model}

In the present work the two-dimensional slab-geometry version of the Hasegawa-Wakatani model is considered. An illustration of the flow configuration is shown in Fig. 1.

The equations in dimensionless form read [2],

$$
\begin{gathered}
\left(\frac{\partial}{\partial t}-\nu \nabla^{2}\right) \nabla^{2} \phi+c(n-\phi)=\left[\nabla^{2} \phi, \phi\right], \\
\left(\frac{\partial}{\partial t}-D \nabla^{2}\right) n+\Gamma \frac{\partial \phi}{\partial y}+c(n-\phi)=[n, \phi],
\end{gathered}
$$

with $n$ the plasma density fluctuation and $\phi$ the electrostatic potential fluctuation. $D$ and $\nu$ are the cross-field diffusion of plasma density fluctuations and kinematic viscosity, respectively. $\Gamma$ can be interpreted as the mean plasma density gradient in the $x$-direction and is taken unity. The adiabaticity $c$, as introduced in the introduction, is related to the parallel dynamics of the plasma,

$c=\frac{T_{e} k_{\|}^{2}}{e^{2} n_{0} \eta \omega_{c i}}$,

with $k_{\|}$the effective parallel wavenumber, $\eta$ the electron resistivity, $T_{e}$ the electron temperature, $e$ the electron charge and $\omega_{c i}$ the ion cyclotron frequency. The equilibrium plasma density is $n_{0}$. The use of an effective parallel wavenumber is of course a simplification, since the fluctuations in the parallel direction are in reality governed by a broad spectrum of parallel wavenumbers, related to the nonlinear cascades in the parallel direction. For a given machine it is however possible that the parallel dynamics are dominated by a particular effective wavenumber $k_{\|}$. The value of $c$, most relevant to describe edge turbulence is therefore not fixed and this should be kept in mind while assessing the results in the present work. Rather than insisting on its physical meaning we use it is as a model parameter which allows to switch between different flow regimes. The Poisson brackets are defined as

$[a, b]=\frac{\partial a}{\partial x} \frac{\partial b}{\partial y}-\frac{\partial a}{\partial y} \frac{\partial b}{\partial x}$.

We identify the $x$-coordinate with the radial direction and the $y$-coordinate with the poloidal direction (cf. Fig. 1). The normalizations are chosen as in the original work in 
which the model was proposed [13] and we will not discuss them further here. Rather will we concentrate on the change in dynamics by evolving the Hasegawa-Wakatani model from its hydrodynamic limit to its geostrophic limit. In order to clearly illustrate the similarities and differences with respect to Navier-Stokes turbulence we rewrite equations $(1,2)$ in two different forms. First we consider the vorticity formulation. The vorticity is defined as $\omega=\nabla^{2} \phi$. The electro-static potential $\phi$ plays thus the role the streamfunction plays in fluid dynamics. Equations $(1,2)$ can then be recast as

$$
\begin{array}{r}
\frac{\partial \omega}{\partial t}+(\boldsymbol{u} \cdot \nabla) \omega=\nu \nabla^{2} \omega-c(n-\phi), \\
\frac{\partial n}{\partial t}+(\boldsymbol{u} \cdot \nabla) n=-\Gamma u+D \nabla^{2} n-c(n-\phi),
\end{array}
$$

in which the velocity $\boldsymbol{u}=\nabla^{\perp} \phi$ :

$u=-\frac{\partial \phi}{\partial y}, \quad v=\frac{\partial \phi}{\partial x}$.

In the Lagrangian dynamics of a fluid particle, in particular in its acceleration, the pressure is known to play a dominant role. The influence of the pressure is not explicitly present in the vorticity formulation. Therefore we recast another time the equations to obtain the velocity formulation. The vorticity is the curl of the velocity so that we have to apply the Biot-Savart operator, which we will denote by $\nabla^{\perp} / \nabla^{2}$, to equation (5), giving

$$
\begin{array}{r}
\frac{\partial \boldsymbol{u}}{\partial t}+(\boldsymbol{u} \cdot \nabla) \boldsymbol{u}=-\nabla p+\nu \nabla^{2} \boldsymbol{u}-\frac{\nabla^{\perp}}{\nabla^{2}}[c(n-\phi)] \\
\nabla \cdot \boldsymbol{u}=0 .
\end{array}
$$

Equation (6) does not change. The pressure appears here as the non-solenoidal part of the Biot-Savart transformed vorticity advection term, which has the role of a Lagrangian multiplier to enforce the incompressibility of the velocity field.

The fluid particle position $\boldsymbol{x}$ at time $t$ is given by the equation

$\frac{d \boldsymbol{x}}{d t}=\boldsymbol{u}(\boldsymbol{x}(t), t)$

in which the velocity is governed by equation (8). The acceleration of a fluid particle is

$\frac{d^{2} \boldsymbol{x}}{d t^{2}}=\frac{\partial \boldsymbol{u}}{\partial t}+(\boldsymbol{u} \cdot \nabla) \boldsymbol{u}$

which should thus be equal to the right hand side of (8). We see that three contributions play a role in the fluid particle acceleration: the pressure gradient, the viscous stresses and the coupling-term proportional to the adiabaticity $c$.

The time-scale-similarity of the Lagrangian dynamics can be conveniently assessed by considering time increments. In the present work we will consider increments of the position of a fluid particle $\delta \boldsymbol{x}(t, \tau)=\boldsymbol{x}(t+\tau)-\boldsymbol{x}(t)$ and of its velocity $\delta \boldsymbol{u}(t, \tau)=\boldsymbol{u}(t+\tau)-\boldsymbol{u}(t)$. Assuming a finite time-correlation for both the position and the velocity, these increments will tend in the limit of large $\tau$ to the sum of two independent variables. In the limit of small $\tau$, we recover the time derivative of the quantity times $\tau$. The shape of the PDF of the position increment at small $\tau$ will therefore tend to the shape of the PDF of the velocity multiplied by the value of $\tau$. Similarly, the shape of the PDF of the Lagrangian velocity increments at small $\tau$ will tend to the PDF of the Lagrangian acceleration times $\tau$.

In the following we will consider four cases with different values of $c$. The smallest value of the adiabaticity is $c=$ 0.01 which should display behaviour close to Navier-Stokes turbulence. The intermediate value $c=0.7$ is generally assumed to be most relevant for the tokamak edge dynamics. The largest values considered here, $c=2$ and 4 , are supposed to display similarities with geostrophic fluid motion.

Starting from random initial conditions, equations $(5,6)$ are integrated using a classical Fourier pseudo-spectral method which is fully dealiased and completed with a semiimplicit time-integration scheme of second order [17]. The resolution is $512^{2}$ and the box-size is $64^{2}$. In Navier-Stokes turbulence the box-size is generally $(2 \pi)^{2}$ in dimensionless units. In the present model length-scales are normalized by the hybrid Larmor-radius $\rho_{s}$ and the size of the box corresponds thus to $64 \rho_{s}$, a value used in previous work $[18,19,5]$. The viscosity and the diffusion coefficient are taken equal to 0.01. The Hasegawa-Wakatani model contains an internal instability, which will lead the flow to a turbulent saturated state, independent of the initial conditions. At $t=300$, the first three cases have reached this turbulent state and for case IV this is at $t=1200$. At that moment $10^{4}$ particles are injected into the system, equally spaced. To solve the particles' advection equation (9), we use a second order Runge-Kutta scheme and the velocity of each particle in a grid-cell is computed via a bicubic interpolation. During a time interval of 300, the particles are stored every ten time steps, one time step being equal to $10^{-3}$.

The Lagrangian acceleration is computed directly by computing the three contributions of equation (8),

$\boldsymbol{a}^{L}=-\nabla p+\nu \nabla^{2} \boldsymbol{u}-\frac{\nabla^{\perp}}{\nabla^{2}}[c(n-\phi)]$,

along the fluid trajectory using again bicubic interpolation to obtain the values at the particle position.

\section{Eulerian Results}

In Figure 2 we show the time-evolution of the kinetic energy and enstrophy. It is observed that after a fast drop, corresponding to the viscous decay of the initial condition, the energy and enstrophy increase and saturate at a certain level. The generation of energy is due to the drift-wave instability, which drains the energy from the imposed plasma density gradient. In equation (6) the $-\Gamma u$ term is responsible for the generation of energy. It corresponds to the gen- 

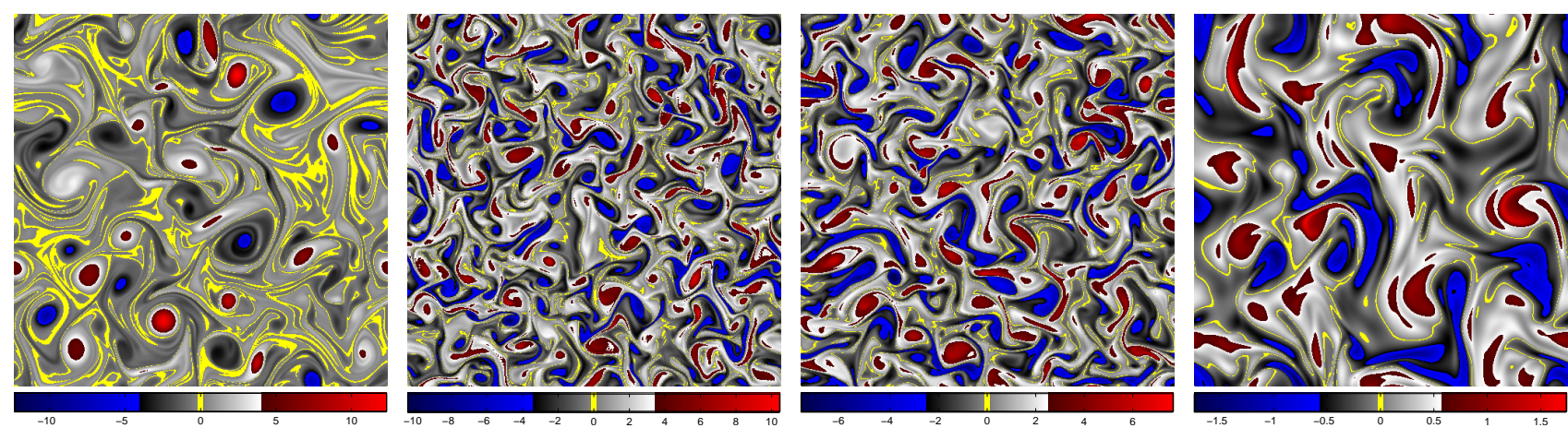

Fig. 3. Visualization of the vorticity field, when a statistically stationary flow regime is established. In this and all following figures, if there are four subfigures, they correspond, from left to right to case I, II, III and IV, respectively.

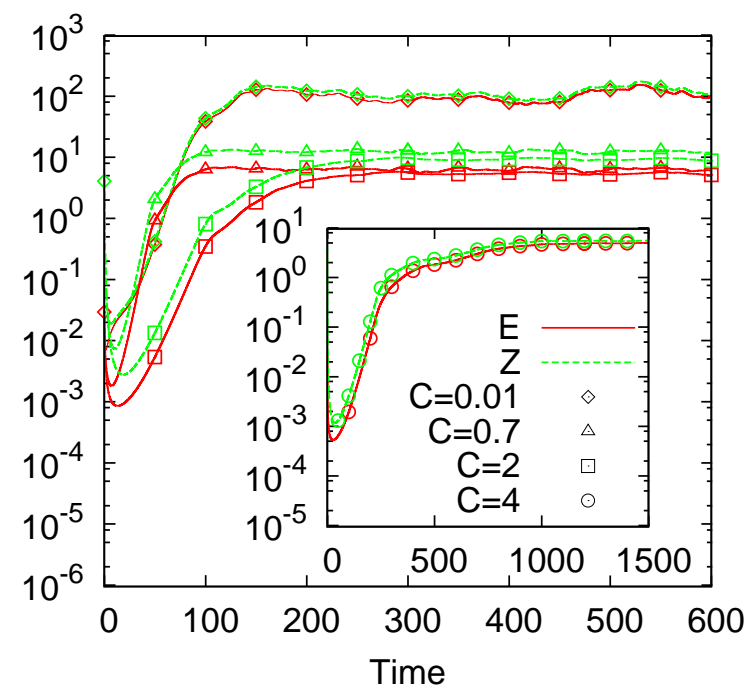

Fig. 2. Kinetic energy and enstrophy as a function of time for case I $(\mathrm{c}=0.01)$, II $(\mathrm{c}=0.7)$, III $(\mathrm{c}=2)$ and, in the inset, IV $(\mathrm{c}=4)$.

eration of plasma density fluctuations through the interaction of the velocity field with the mean gradient $\Gamma$. The variance of the plasma density fluctuations is then transferred though non-linear interaction among wavenumbers and serves as an energy input in the velocity field through the coupling term $c(n-\phi)$. At long times statistical equilibrium is reached between the source term and the nonlinear and viscous processes. It is also observed that this time for $c=4$ is significantly later than for the other three cases. This is related to the tendency of the Hasegawa-Mima system to transfer energy to the larger scales by means of the inverse cascade [20]. An alternative explanation is that the linear growth rate of the Hasegawa-Wakatani system is a decreasing function of $c$, as proposed in [21]. In this work one can also find a detailed study of the transfer mechanisms between the different wavemodes and the different fields.

In the flow visualizations, Figure 3, it can be seen that the flow structures for the $c=0.01$ and $c=4$ case are larger than for the two intermediate cases. In the case $c=$ 0.01 this is because the energy is mainly injected into the small wavenumbers. Indeed, to a first approximation, the drift-wave instability will inject most of its energy around the peak of the growth rate spectrum of the most unstable mode. This peak is an increasing function of $c$ [18]. The main energy injection for the $c=4$ case is thus at larger wavenumbers then for the case $c=0.01$. However, through the inverse energy cascade large structures are formed on a larger time-scale. The slowly increasing energy between $t=300$ and $t=800$ corresponds to the time-interval in which the inverse cascade builds increasingly larger flow structures. Apparently, in the two intermediate cases this inverse energy cascade is absent, so that the flow-structures are smaller.

The isotropic energy spectra in Figure 4 illustrate the energy distribution in Fourier space. It is observed that the energy is peaked at smaller wavenumbers for the cases $c=$ 0.01 and $c=4$ than for the two intermediate cases, which reflects the larger structures observed in case I and IV. The spectra exhibit power-law scaling with an exponent close to -4 , which is typically observed in forced two-dimensional Navier-Stokes turbulence in the forward enstrophy range. Indeed, the scaling close to the energy injection generally displays power-laws which are steeper than the -3 Kraichnan prediction [22], due to logarithmic corrections [23]. The $-5 / 3$ inverse cascade seems absent. This can be understood since the coupling term acts as a friction term, which drains energy from the velocity field at the smallest wavenumbers. In the present case, the region where the friction is dominant coincides approximately with the region where the inverse cascade is expected.

In table 1, typical parameters characterizing the turbulence and the turbulent transport are given for the simulations. The correlation coefficient $\rho_{u n}=\overline{u n} / \sqrt{\overline{u^{2}} \overline{n^{2}}}$ characterizes the efficiency of the flow to transport plasma density fluctuations. The normalized turbulent flux is most efficient for intermediate values of $c$. This result was also found in Camargo et al. [21]. For large values, the wavy character of the velocity does not well transport the density. Intuitively this is understandable since pure waves will simply oscillate the fluid particles. The case of very coherent structures in the limit of small $c$ is not most efficient either. Indeed, 


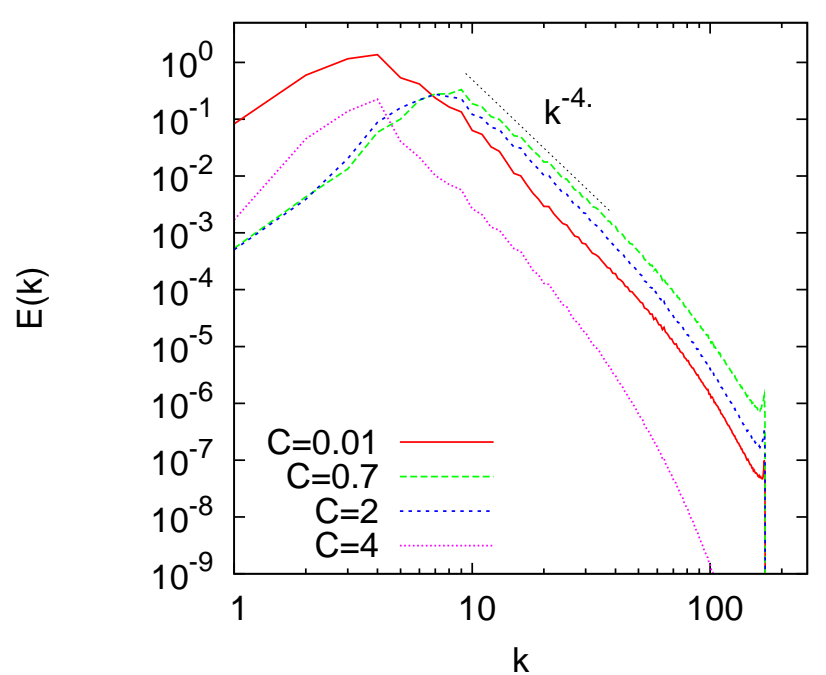

Fig. 4. Kinetic energy spectra. The maximum of the energy spectra of case I and IV is at smaller wavenumbers than case II and III. The slope $k^{-4}$ is plotted for reference.

\begin{tabular}{c||c|c|c|c}
\hline \hline $\mathrm{c}$ & 0.01 & 0.7 & 2 & 4 \\
\hline$\overline{u^{2}}$ & 4.1 & 1.9 & 1.6 & 0.42 \\
$\overline{v^{2}}$ & 3.9 & 1.7 & 1.4 & 0.38 \\
$\overline{n^{2}}$ & 162 & 6.9 & 6.0 & 7.3 \\
$\overline{u n}$ & 5.2 & 0.94 & 0.37 & 0.017 \\
$\overline{v n}$ & 0.021 & 0.028 & $1.0 \cdot 10^{-3}$ & $1.1 \cdot 10^{-3}$ \\
$\rho_{u n}$ & 0.20 & 0.26 & 0.12 & $9.7 \cdot 10^{-3}$ \\
$\rho_{v n}$ & $8.4 \cdot 10^{-4}$ & $8.1 \cdot 10^{-3}$ & $3.5 \cdot 10^{-4}$ & $6.7 \cdot 10^{-4}$ \\
\hline \hline
\end{tabular}

Table 1

Average values of some typical turbulence quantities. These quantities are averaged over space and time during a time-interval of approximately 300 time units. The correlation coefficient $\rho_{u n}$ is defined as $\rho_{u n}=\overline{u n} / \sqrt{\overline{u^{2}} \overline{n^{2}}}$ and analogous for $\rho_{v n}$.

trapped fluid particles in a fixed coherent structure will on average contribute little to the flux. This effect (see also the work by Koniges et al. [18]) can be described as a function of the Kubo-number [24], defined as the ratio of the correlation time of the velocity field over the sweeping time, associated to the large-scale velocity and length-scale of the flow (see e.g. references [25-27]).

\section{Lagrangian Results}

\subsection{Trajectories and single particle dispersion}

In Figure 5 the trajectories of the fluid particles are plotted. It is clear again that the flow-structures in regime I are larger than in case II and III. This is less pronounced for case IV. Another feature is the anisotropy, which is important at large values of $c$. In particular in the case $c=4$ a clear vertical particle drift is observed. This drift gives rise to a super-diffusive dispersion as can be seen in Figure 6,

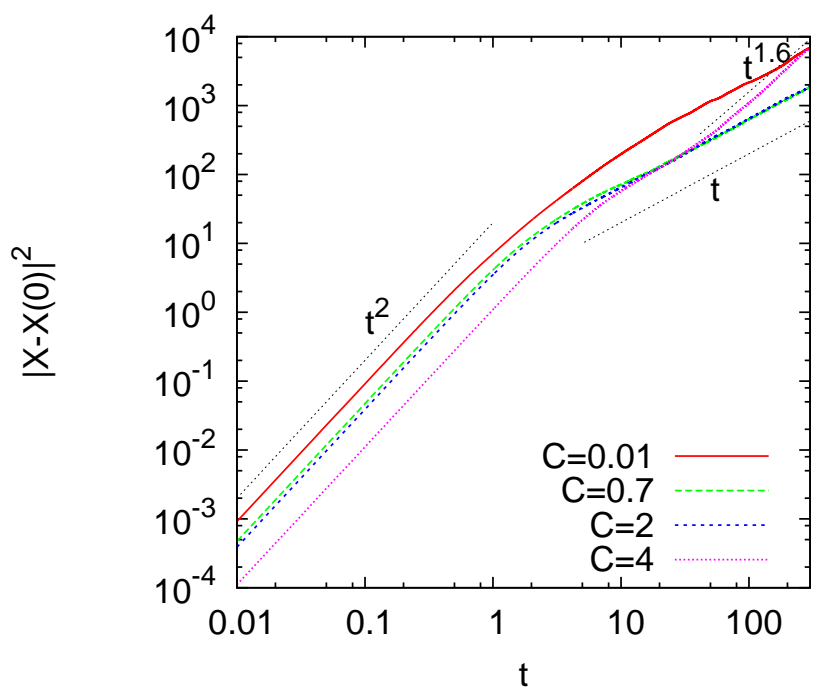

Fig. 6. Single particle dispersion. The ballistic and brownian behaviour are present in all cases. In case IV a long-time super-diffusive behaviour is observed.

in which $|X-X(0)|$ is the displacement of a fluid particle from its initial position. For short times the dispersion is ballistic and and for larger times Brownian, which corresponds to decorrelated motion. Only in the case $c=4$ for the longest times a superdiffusive behaviour is observed, corresponding to the non-random vertical plasma drift. Indeed, movies show a clear, vertical drift velocity, which is approximately uniform. At long times, this drift would induce a $t^{2}$ dependence of the displacement from the initial position. The anisotropy of the dispersion was discussed in [28]. The drift is not confined to a radially localized band (even though the computational domain itself represents only a small segment of a tokamak), so that it is not clear whether it can be defined as a zonal flow [29]. The influence of the Reynolds number on these flows also necessitates further study. Indeed, in a recent theoretical study it was proposed that the structure of the dissipative mechanisms is directly related to the strength of the zonal flows in drift-wave turbulence [30]. However, it seems more probable that the flow is a function of collisional drag, acting at all scales, than that the viscous and diffusive effects, dominant at the smallest scales, play a role.

\subsection{PDFs of time increments: a note on the definition of Lagrangian Intermittency}

In the insets of Figure 7 the PDF of the positionincrements is shown for the different cases. In case II, III and IV the PDF at both small and large $\tau$ is Gaussian. In Figure 7 we also show the PDF of the absolute value of the position-increments. As it is supposed to be for the norm of a Gaussian-distributed quantity, the PDF obeys a Rayleigh-distribution. The step size in the tokamak relevant regime is thus not intermittent, contrarily to what was stated in [5]. For the case $c=0.01$, all distributions are 

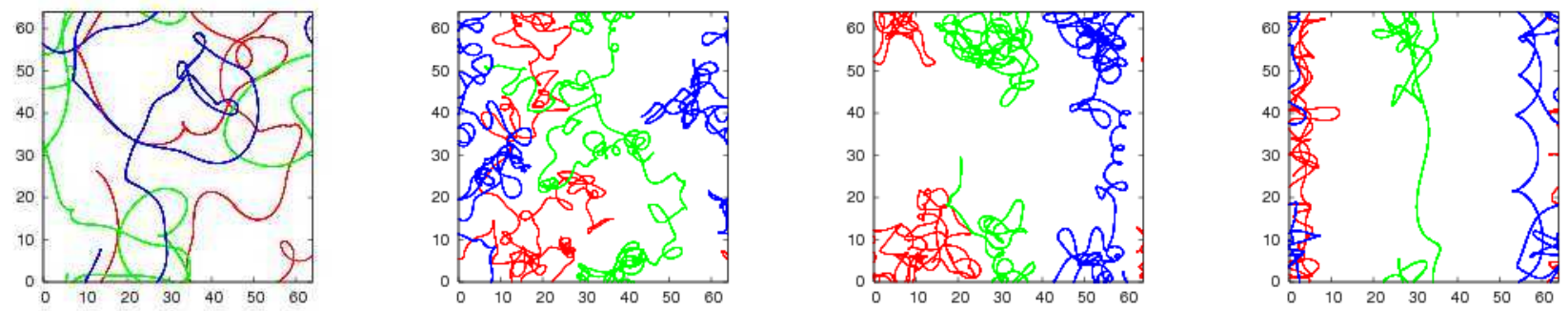

Fig. 5. Particle trajectories. In case I the spiraling motion corresponds to the larger flow structures than in case II and III. Clear drift-trajectories are observed for case IV.
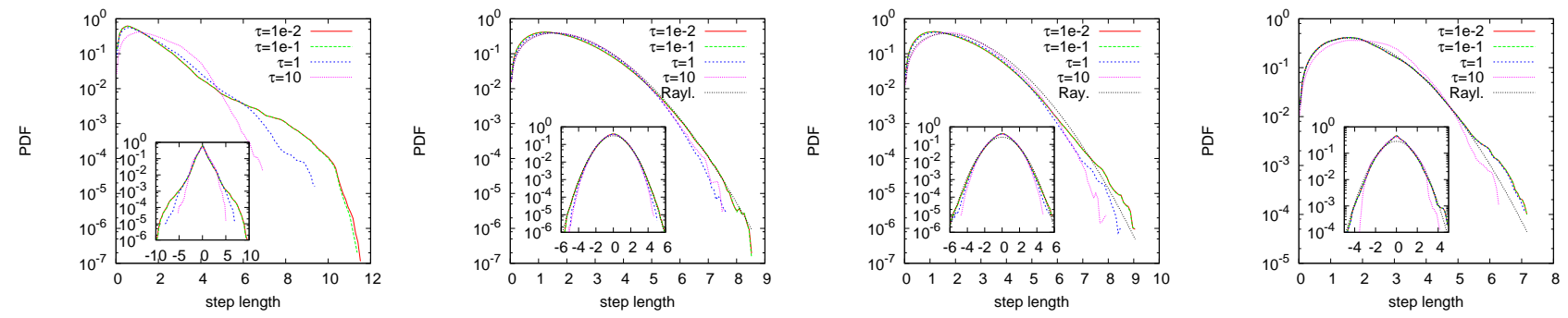

Fig. 7. Step length PDFs. The PDFs of the norm of the particle position increments is shown. In the inset the PDF of the value (not the norm) is shown. All PDFs in this paper are normalized by their corresponding standard deviation.

strongly non-Gaussian. This regime, even though it shares some similarities with hydrodynamic turbulence, is not completely equivalent since in hydrodynamic turbulence the velocity PDF is generally Gaussian.

In Figure 8 the PDF of the velocity-increments is shown for the different cases. In case I the PDF is non-Gaussian at large $\tau$ and becomes increasingly non-Gaussian with decreasing $\tau$. We note that in [31] similar PDFs were observed, where the non-Gaussianity was related to long-living coherent structures. The Lagrangian intermittency is clearly present in the sense that the flaring tails become increasingly wider at smaller time-scales. Regime II, III and IV show PDFs which evolve from a Gaussian to a Laplace (or exponential) distribution. This is expected if the acceleration is governed by the pressure-gradient contribution. As was shown by Holzer and Siggia [16], the quadratic nonlinearity in the Navier-Stokes equations leads to an exponential distribution for the pressure gradient if the velocity field is a Gaussian, non intermittent field. This is also discussed in [32] and a similar argument can be found in [33]. If the pressure gradient is the dominant term in the acceleration (equation (11)), the acceleration (and the small $\tau$ velocity increment PDF) are expected to be exponential.

This has an important implication for the definition of intermittency: if the presence of intermittency is detected by the change in shape of the velocity increment PDF, then Lagrangian statistics are always intermittent because the pressure gradient, which dominates the increments at small $\tau$, is exponential due to the quadratic dependence of the pressure on the velocity even in non-intermittent fields. The definition of intermittency should therefore in our opinion be modified. A more adequate definition of intermittency in the Lagrangian framework should read: if the shape of the PDF changes and its flatness for small $\tau$ is superior to 6 (the value of the flatness of the Laplace distribution), the characteristics are intermittent.

Using this modified definition we arrive at one of the main observations of the present work: from a Lagrangian point of view, the Hasegawa-Wakatani model in the quasiadiabatic regime is not intermittent. The flatness of the velocity increment PDFs as a function of the time increment $\tau$ is displayed in Figure 9. In the hydrodynamic case the flatness decreases from values superior to 50 at small $\tau$ to the Gaussian value 3 at long times. The flatness of the Laplace distribution is 6. Cases II, III and IV show values in between 5.5 and 9 for small $\tau$, which is close to the theoretical value for non-intermittent velocity fields.

We highlight this in Figure 9, where all the acceleration PDFs of regime II,III and IV collapse perfectly and can be fitted by a Laplace distribution, while regime I shows large flaring tails. A question which one can ask is if there is a sharp threshold between intermittent and non-intermittent behavior. To check this we performed a supplementary computation with $c=0.1$. The acceleration PDFs are shown in figure 10. Indeed the PDF for $c=0.1$ shows tails intermediate between the exponential tails for large $c$ and the heavy tails for $c=0.01$. The transition between intermittent and non-intermittent dynamics seems thus to be smooth. For the largest values of the acceleration, the tails fall off rapidly. It was checked that this is not a problem of statistical convergence. It is however not clear whether this fall-off is a physical effect or an artefact of the numerical simulations. 

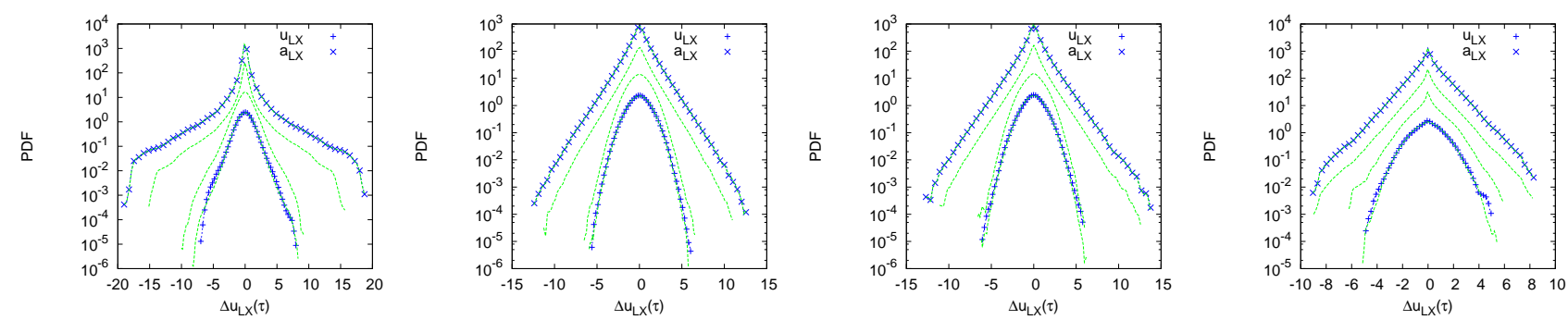

Fig. 8. PDFs of the Lagrangian velocity increments. At large $\tau$ the PDFs are close to Gaussian and for smaller $\tau$ the PDFs become increasingly non-Gaussian. PDFs are shifted vertically for clarity.
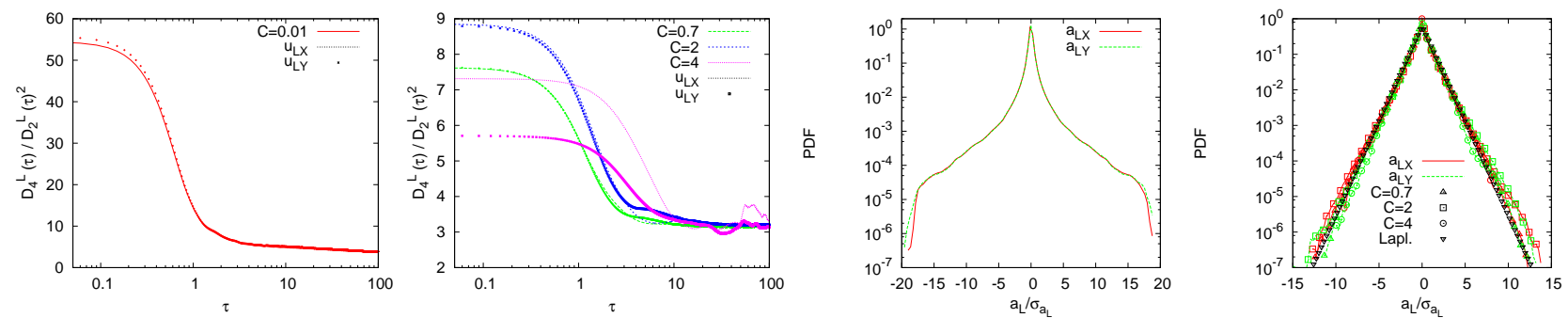

Fig. 9. Left two graphs: flatness of the Lagrangian velocity increments PDFs as a function of $\tau$. Right two graphs: acceleration PDFs. The PDFs of case II, III and IV collapse and can be fitted by a Laplace distribution, indicated by black symbols.

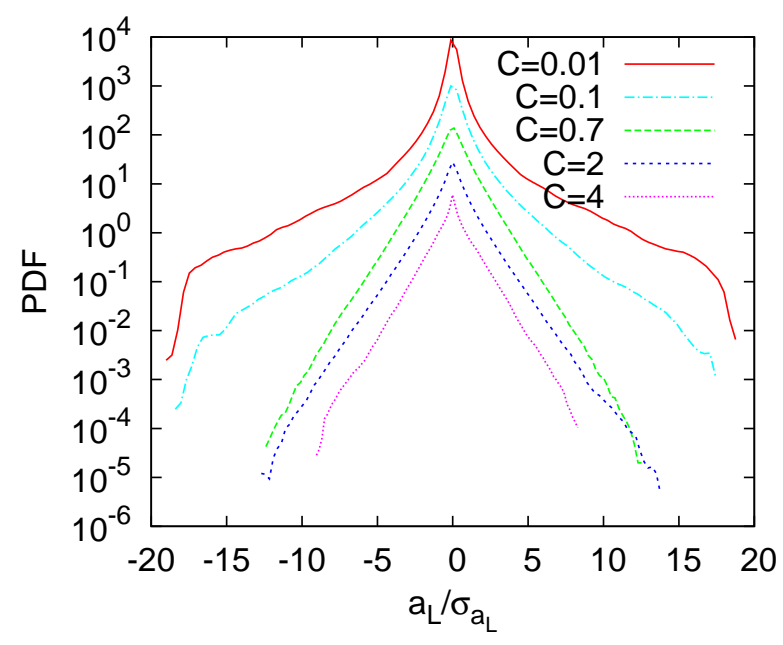

Fig. 10. Comparison of the PDFs of the $x$-component of the acceleration for 5 different values of $c$. PDFs are shifted vertically for clarity.

\subsection{Lagrangian structure functions: a note on the use of Extended Self-Similarity}

Lagrangian structure functions of the order $p$ are defined as

$D_{p}^{L}(\tau)=\left\langle|u(t+\tau)-u(t)|^{p}\right\rangle_{t}$

in which $\langle\cdot\rangle_{t}$ represents an average over $t$. According to K41like dimensional arguments, in the inertial range $D_{p}^{L}(\tau)$ should scale as

$D_{p}^{L}(\tau) \sim(\epsilon \tau)^{\zeta_{p}^{L}}$ with $\zeta_{p}^{L}=p / 2$. The deviation of these exponents reflects the Lagrangian intermittency. In practice the inertial ranges are very small, even more in the Lagrangian than in the Eulerian framework so that a precise determination is not simple. This explains the success of Extended SelfSimilarity (ESS) [34]. ESS is a widely used tool, which amplifies the scaling range over which power-laws are observed. An important question is whether these wide ranges correspond to inertial ranges. In the Lagrangian context ESS consists in plotting $D_{p}^{L}(\tau)$ as a function of $D_{2}^{L}(\tau) . D_{2}^{L}(\tau) \sim \tau$ according to K41-like arguments, and it is shown that the scaling of the Lagrangian structure functions holds over a wider range in this representation. This was illustrated in e.g. [35].

In Figure 11 we show the ESS representation of the structure-functions of orders $p=3-8$. The straight lines are the non-intermittent prediction $\zeta_{p}^{L}=p / 2$. At a first glance this prediction holds. A first, uncareful, conclusion could be drawn that all structure functions exhibit K41 scaling. One should however not forget the following. For very short timelags $\tau$ one can, using Taylor series, write

$$
\begin{aligned}
D_{p}^{L}(\tau) & =\left\langle|u(t+\tau)-u(t)|^{p}\right\rangle_{t} \\
& \approx\left\langle\left|\frac{d u(t)}{d t} \tau\right|^{p}\right\rangle_{t} \\
& =\left\langle\left|a^{L}\right|^{p} \tau^{p}\right\rangle_{t} \\
& =\left\langle\left|a^{L}\right|^{p}\right\rangle_{t} \tau^{p} \\
& \sim \tau^{p}
\end{aligned}
$$

Note that the error of this approximation is of order $\tau^{p+1}$ and hence becomes smaller for increasing values of $p$. From 

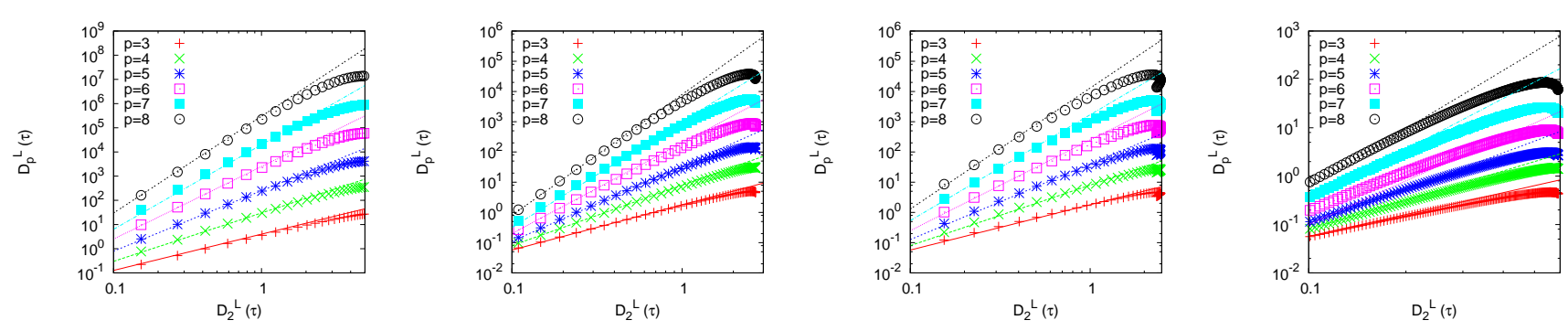

Fig. 11. ESS analysis of the Lagrangian structure functions of order 3 to 8 . The straight lines correspond to K41 predictions.

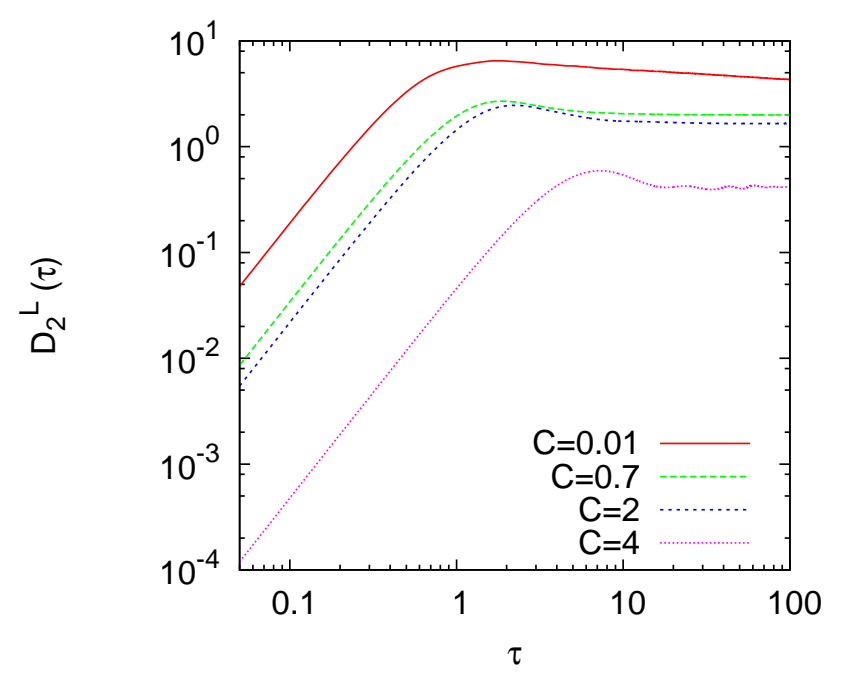

Fig. 12. $D_{2}^{L}(\tau)$, the second order Lagrangian structure function.

(18) trivially follows:

$D_{p}^{L}(\tau) \sim D_{2}^{L}(\tau)^{p / 2}$

Hence at short time intervals, shorter than the Lagrangian acceleration correlation time, ESS yields always and trivially K41 scaling. This explains why K41 was observed in [35] for times shorter than the Kolmogorov time. Is it possible to determine the inertial range scaling? This is questionable as is illustrated in Figure 12, where we show the second order structure function $D_{2}^{L}(\tau)$. The scaling as predicted by foregoing arguments for small times is clearly present. The $\tau^{2}$ range extends over more than one decade and can even be extended indefinitely if $\tau$ is decreased. To observe the expected inertial range slope proportional to $\tau$, one needs some imagination. This range, if present, is very small. We will therefore not intend to determine the scaling exponents of the structure functions in the present work. Larger Reynolds numbers would be needed to determine them.

The Lagrangian energy spectrum, obtained from Fouriertransforming the Lagrangian velocity data along the trajectories, is shown in Figure 13. In the hydrodynamic regime a scaling range can be observed, which is larger than in the second order structure functions. The inertial range is proportional to $f^{-2}$, as observed in previous works [9]. In the inset the acceleration spectrum is shown, which should be similar to the velocity spectrum compensated by $f^{2}$. The acceleration spectrum shows a small but clear plateau. In the other 3 regimes this plateau is not visible.

\subsection{Time-auto-correlations: a note on long-time correlations}

In [36] it was proposed that long time-correlations of the norm of the acceleration are a key-feature of Lagrangian intermittency. Indeed, the direction of the velocity of fluid particles in a turbulent velocity field changes rapidly so that the Cartesian components of the acceleration vector decorrelate on a short time scale, typically of the order of the Kolmogorov time-scale. The norm of the acceleration remains correlated over time-scales of the order of the eddy turn over time, since the acceleration is dominated by the centripetal component, when the fluid particles follow a vortical motion and because fluid particles remain trapped in vortices for relatively long times.

In Figure 14 we show the velocity auto-correlations and the acceleration auto-correlations for the 4 regimes. These correlations are shown for both $x$ and $y$ components and for the norm. One recurrent feature in all the regimes is that the norm of the acceleration (and the norm of the velocity) is correlated over longer times than the components of the acceleration. Even in the non-intermittent cases II, III and IV this is the case. It would be interesting to check whether there exists a quantitative link between the length of the time-correlations and the flatness of the acceleration PDFs. A detailed investigation of this will be postponed to a future study. This would allow to check the statement in [36] that long time-correlations are a key to Lagrangian intermittency.

\subsection{A note on intermittency models}

We showed in the previous sections that drift-wave turbulence is not intermittent in the Lagrangian framework for $c \geq 0.7$. A question is now how we can reconcile this with recent work claiming that the case $c=0.7$ is intermittent. In this section we will try to understand the difference in the reasoning between [5] and the present work. First, on which observation do they base their conclusion that the flow is intermittent? In [5] structure functions were determined for various quantities in the Hasegawa-Wakatani 

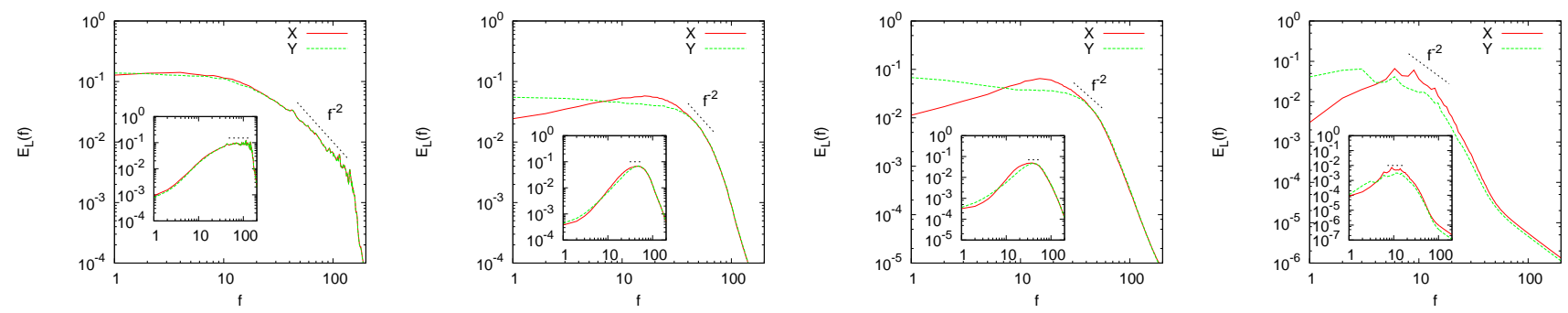

Fig. 13. Lagrangian energy spectra. The insets show the acceleration spectrum which can be obtained by compensating the spectra by $f^{2}$.
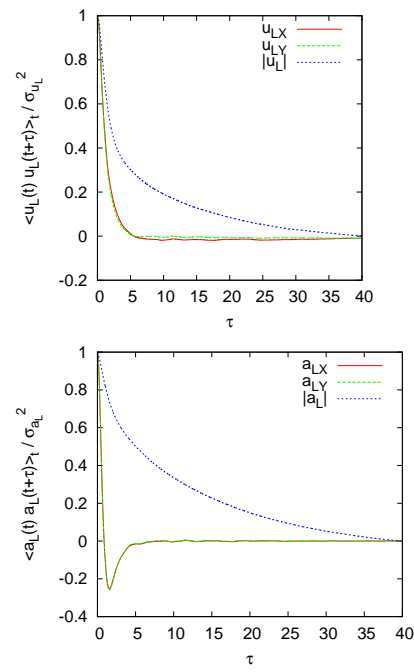
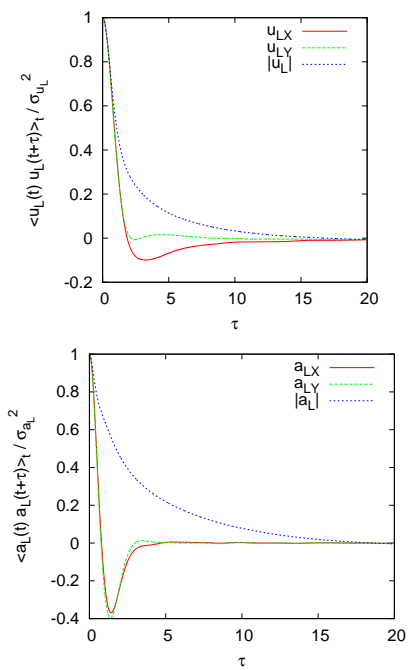
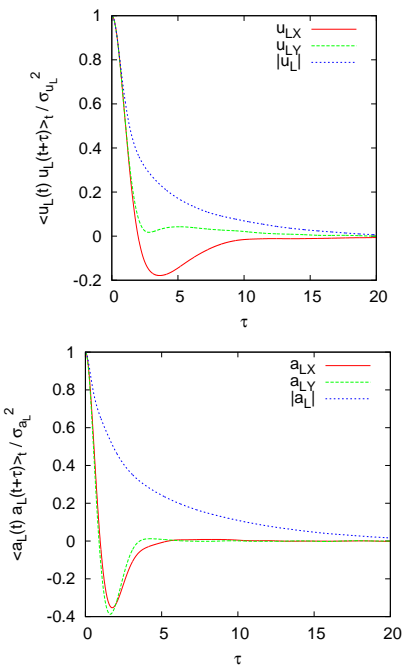
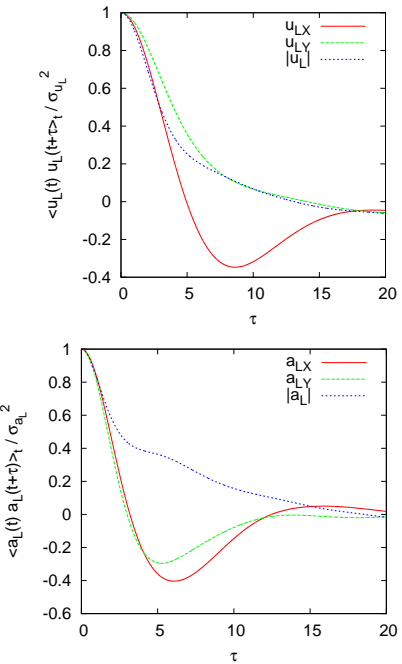

Fig. 14. Lagrangian velocity auto-correlations and acceleration auto-correlations for both $x$ and $y$ components and for the norm.

model. Note that they considered spatial velocity increments, which assess the Eulerian scale-self-similarity. However, generally, Lagrangian intermittency is stronger in the Lagrangian framework $[9,10]$, so that this difference does not explain the discrepancy between the conclusions. In [5] it is shown that the velocity increment structure functions scale perfectly well according to K41 arguments, i.e., the values of the scaling exponents are $p / 3$, which corresponds to a velocity field free from intermittency. Subsequently they determine the scaling exponents of the vorticity increments and they compare the results to K41 predictions for the velocity. They show that the scaling exponents are close to the She-Leveque model [37], which is a model which is able to fit the exponents for the velocity-increment structure functions. We think that the agreement is fortituous. A possible explanation of the results will be given here.

In 1994, the same year that She and Leveque proposed their model, Kraichnan [38] showed that a passive scalar shows anomalous scaling exponents, even when the advecting velocity field is completely Gaussian, solenoidal and $\delta$ correlated in time. In figure 15 we show the exponents predicted by this model, compared to the She-Leveque exponents. The values are very close and within numerical or experimental errors hard to distinguish. We think that this is the real explanation for the observations in [5]: the 'anomalous' scaling of the vorticity structure functions is related to the anomalous scaling of a passive scalar. It was shown in recent work [4] that the scalar behaviour is close to the behaviour of the vorticity in the case considered $(c=0.7)$. This explains that the vorticity agrees with the same scaling. However the interpretation that the dissipative structures in drift-wave turbulence are vorticity filaments does not follow from these results.

\section{Conclusion}

We presented a detailed Lagrangian investigation of dissipative drift-wave turbulence in the Hasegawa-Wakatani model. This model is generally assumed to possess some similarities with tokamak-edge plasma-turbulence. One of the goals was to characterize the Lagrangian intermittency of this kind of micro-turbulence. We showed that within the present model, for $c \geq 0.7$, drift-wave turbulence is not intermittent. We also gave an explication why in recent work it was thought that this kind of turbulence is intermittent [5]. The above observations suggest to model the transport in drift-wave turbulence, except for the hydrodynamic limit, by a simple Langevin-like stochastic process.

From a point of view of fluid mechanics, the present investigation is also interesting. The continuous change of behaviour between a quasi-hydrodynamic and a quasigeostrophic flow regime allows to highlight the differences of 


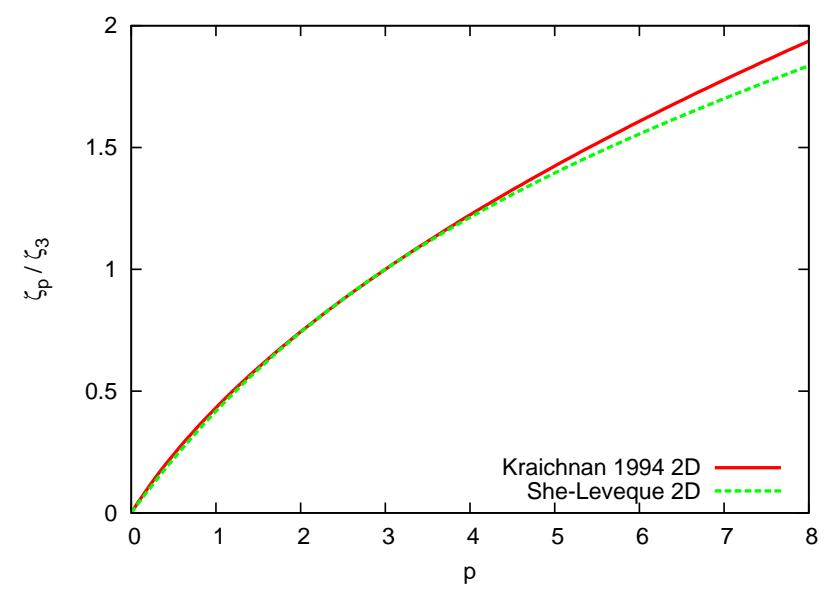

Fig. 15. Comparison of the She-Leveque model and the Kraichnan prediction. Even though the curves are very similar, they have been derived for different quantities: She-Leveque for the velocity structure functions, Kraichnan's model for the passive scalar structure functions.

the different flows, within the same framework. We hereby showed that the Lagrangian intermittency, characterized by flaring tails disappears when the model parameter $c$ is increased. Indeed all the PDFs of the Lagrangian acceleration for $c \geq 0.7$ collapse on a Laplace distribution, which is not a sign of intermittency.

To conclude, we resume our findings.

(i) Drift-wave turbulence is not intermittent in the Hasegawa-Wakatani model for $c \geq 0.7$. We demonstrate this in the Lagrangian framework and we show how, in previous work [5], the Eulerian analysis also supports this. For smaller values of $c$, the exponential tails of the acceleration PDFs gradually become stretched exponentials.

(ii) We argue that the change of shape of the velocityincrement PDFs is not an adequate indicator for intermittent behaviour. Indeed for Gaussian nonintermittent velocity fields the PDF of the increments changes its shape from a Gaussian distribution at long $\tau$ to a Laplace distribution at small $\tau$. Intermittency could be detected if the tails of the PDF are heavier than exponential.

(iii) We show that ESS might be a dangerous tool, because it induces K41 scaling for small $\tau$ in a trivial way, unrelated to inertial range behaviour. This feature explains the experimental observation [35] that K41 scaling is observed for time increments smaller than the Kolmogorov time.

\section{Perspectives}

Several questions remain unanswered in the present investigation and require further research. One is the issue of the Reynolds number. It should be checked what the influence of the viscosity is on the PDF of the Lagrangian acceleration. Indeed, it was shown in simulations and exper- iments of three-dimensional fluid turbulence that the tails of the acceleration PDFs are a function of the Reynolds number $[39,40]$. For the moment we cannot exclude that the non-intermittent character of the Lagrangian velocity increments in the large $c$ cases will become more intermittent at larger Reynolds numbers.

Future work could also focus on the poloidal drift velocity observed at large values of $c$. Indeed in a recent theoretical study [30], it was proposed that the zonal flow is directly proportional to the inverse of the collisional drag.

Another issue which should be checked quantitatively is the relation between the long-time correlations of the norm of the acceleration on the intermittent form of the acceleration PDFs. Indeed, it seems that for large $c$ the fluid particle dynamics can be described by a random walk. For small $c$ a multifractal random walk, as proposed in [36] might better describe the dynamics.

We hope that these insight inspire the development and improvement of Lagrangian models for turbulent transport in the tokamak edge.

\section{Acknowledgments}

We thank S. Benkadda and S. Futatani for introducing us to the Hasegawa-Wakatani system. We thank M. Bourgoin for a discussion on Lagrangian structure functions and Wendel Horton for comments on the manuscript. Two anonymous referees are acknowledged for comments which significantly improved the present paper. We thankfully acknowledge financial support from the Agence Nationale pour la Recherche, project 'M2TFP'. S.N. and K.S. thank the Deutsch-Französische Hochschule, project 'S-GRKED-04-05', for financial support.

\section{References}

[1] F. Toschi and E. Bodenschatz. Lagrangian properties of particles in turbulence. Annu. Rev. Fluid Mech., 41:375, 2009.

[2] W. Horton. Nonlinear drift waves and transport in magnetized plasma. Phys. Rep., 192:1, 1990.

[3] B. D. Scott. The nonlinear drift wave instability and its role in tokamak edge turbulence. New J. Phys., 4:52, 2002.

[4] M. Priego, O. E. Garcia, V. Naulin, and J. Juul Rasmussen. Anomalous diffusion, clustering, and pinch of impurities in plasma edge turbulence. Phys. Plasmas, 12:062312, 2005.

[5] S. Futatani, S. Benkadda, Y. Nakamura, and K. Kondo. Characterization of intermittency of impurity turbulent transport in tokamak edge plasmas. Phys. Plasmas, 15:072506, 2008.

[6] P.K. Yeung and S.B. Pope. Lagrangian statistics from direct numerical simulations of isotropic turbulence. J. Fluid Mech., 207:531, 1989.

[7] S. Ott and J. Mann. An experimental investigation of the relative diffusion of particle pairs in three-dimensional turbulent flow. J. Fluid Mech., 422:207, 2000.

[8] A.N. Kolmogorov. The local structure of turbulence in incompressible viscous fluid for very large Reynolds numbers. Dokl. Akad. Nauk. SSSR, 30:301-305, 1941. 
[9] N. Mordant, P. Metz, O. Michel, and J.-F. Pinton. Measurement of lagrangian velocity in fully developed turbulence. Phys. Rev. Lett., 87:214501, 2001.

[10] T. Ishihara, Y. Kaneda, M. Yokokawa, K. Itakura, and A. Uno. Small-scale statistics in high-resolution direct numerical simulation of turbulence: Reynolds number dependence of onepoint velocity gradient. J. Fluid Mech., 592:335, 2007.

[11] O. Kamps and R. Friedrich. Lagrangian statistics in forced twodimensional turbulence. Phys. Rev. E, 78:036321, 2008.

[12] B. Kadoch, W.J.T. Bos, and K. Schneider. Extreme lagrangian acceleration in confined turbulent flow. Phys. Rev. Lett., 100:184503, 2008.

[13] A. Hasegawa and M. Wakatani. Plasma edge turbulence. Phys. Rev. Lett., 50:682, 1983.

[14] J.G. Charney. On the scale of atmospheric motions. Geofys. Publ., 17:1, 1948.

[15] A. Hasegawa and K. Mima. Pseudo-three-dimensional turbulence in magnetized nonuniform plasma. Physics of Fluids, 21:87, 1978 .

[16] M. Holzer and E. Siggia. Skewed, exponential pressure distributions from Gaussian velocities. Phys. Fluids A, 5:2525, 1993.

[17] K. Schneider and M. Farge. Decaying two-dimensional turbulence in a circular container. Phys. Rev. Lett., 95:244502, 2005

[18] A. E. Koniges, J. A. Crotinger, and P. H. Diamond. Structure formation and transport in dissipative drift-wave turbulence. Phys. Fluids B, 4:2785, 1992.

[19] W.J.T. Bos, S. Futatani, S. Benkadda, M. Farge, and K. Schneider. Role of coherent vorticity in turbulent transport in resistive drift-wave turbulence. Phys. Plasmas, 15:072305, 2008.

[20] D. Fyfe and D.C. Montgomery. Possible inverse cascade behavior for drift-wave turbulence. Phys. Fluids, 22:246, 1979.

[21] S. J. Camargo, D. Biskamp, and B. D. Scott. Resistive driftwave turbulence. Phys. Plasmas, 2:48, 1995.

[22] R.H. Kraichnan. Inertial ranges in two-dimensional turbulence. Phys. Fluids, 10:1417, 1967.

[23] R.H. Kraichnan. Inertial-range transfer in a two- and a threedimensional turbulence. J. Fluid Mech., 47:525-535, 1971.

[24] R. Kubo. Stochastic Liouville equations. J. Math. Phys., 4:174, 1963.

[25] P. Castiglione. Diffusion coefficients as function of Kubo number in random fields. J. Phys. A, 33:1975, 2000.

[26] M. Vlad, F. Spineanu, J. H. Misguich, J. D. Reuss, R. Balescu, K. Itoh, and S. I. Itoh. Lagrangian versus Eulerian correlations and transport scaling. Plasma Phys. Control. Fusion, 46:1051, 2006

[27] T. Hauff and F. Jenko. Turbulent E x B advection of charged test particles with large gyroradii. Phys. Plasmas, 13:102309, 2006.

[28] V. Naulin and J.J. Rasmussen. Aspects of turbulence transport. Contrib. Plasma Phys., 44:546, 2004.

[29] P.H. Diamond, S.-I. Itoh, K. Itoh, and T.S. Hahm. Zonal flows in plasma - a review. Plasma Phys. Control. Fusion, 47:35, 2005.

[30] P.H. Diamond, O.D. Gurcan, T.S. Hahm, K. Miki, Y. Kosuga, and X. Garbet. Momentum theorems and the structure of atmospheric jets and zonal flows in plasmas. Plasma Phys. Contr. Fusion, 50:124018, 2008.

[31] T. Dubos, A. Babiano, J.Paret, and P. Tabeling. Intermittency and coherent structures in the two-dimensional inverse energy cascade: Comparing numerical and laboratory experiments. Phys. Rev. E, 64:036302, 2001.

[32] K. Yoshimatsu, N. Okamoto, K. Schneider, Y. Kaneda, and M. Farge. Intermittency and scale-dependent statistics in fully developed turbulence. Phys. Rev. E, 79:026303, 2009

[33] A. Tsinober, P. Vedula, and P. K. Yeung. Random taylor hypothesis and the behavior of local and convective accelerations in isotropic turbulence. Phys. Fluids, 13:1974, 2001.
[34] R. Benzi, S. Ciliberto, R. Tripiccione, C. Baudet, F. Massaioli, and S. Succi. Extended self-similarity in turbulent flows. Phys. Rev. E, 48:R29, 1993.

[35] H. Xu, M. Bourgoin, N.T. Ouellette, and E. Bodenschatz. High order Lagrangian velocity statistics in turbulence. Phys. Rev. Lett., 96:024503, 2006.

[36] N. Mordant, J. Delour, E. Leveque, A. Arneodo, and J.-F. Pinton. Long time correlations in Lagrangian dynamics: a key to intermittency in turbulence. Phys. Rev. Lett., 89:254502, 2002.

[37] Z.-S. She and E. Leveque. Universal scaling laws in fully developed turbulence. Phys. Rev. Lett., 72:336, 1994.

[38] R.H. Kraichnan. Anomalous scaling of a randomly advected passive scalar. Phys. Rev. Lett., 72:1016, 1994.

[39] P. Vedula and P. K. Yeung. Similarity scaling of acceleration and pressure statistics in numerical simulations of isotropic turbulence. Phys. Fluids, 11:1208, 1999.

[40] G.A. Voth, A. La Porta, A.M. Crawford, J. Alexander, and E. Bodenschatz. Measurements of particle accelerations in fully developed turbulence. J. Fluid Mech., 469:121, 2002. 